saccades $>100 \mathrm{deg} / \mathrm{s}$, and head movements. MRI examinations were performed at $2.5-5$ years of age, including ADC measurements at multiple locations.

Results There were significant univariate correlations between some gaze parameters, MR abnormalities and asymmetries in ADC values. Moreover, there was a correlation between the number of observed perivascular spaces and SP $\left(r_{\mathrm{s}}=-0.407 \mathrm{p}=\right.$ $0.025)$

Conclusion This is the first study to examine the relationship between young infants' visual tracking abilities and later MRI. Poorer tracking for gaze and saccades correlated with white matter damage, while SP correlated with ADC values for the pons.

\section{PO-0429 2-YEAR-OUTCOME OF SURVIVING PRETERM INFANTS (GESTATIONAL AGE 23-25 WEEKS) IN SWITZERLAND: IMPACT OF THE 2002/SWISS GUIDELINES FOR THE CARE OF INFANTS BORN AT LIMIT OF VIABILITY}

${ }^{1} \mathrm{G}$ Natalucci, ${ }^{2} \mathrm{~B}$ Latal, ${ }^{3} \mathrm{HU}$ Bucher, ${ }^{4} \mathrm{TM}$ Berger, ${ }^{3} \mathrm{M}$ Adams, ${ }^{5}$ for the Swiss Neonatal Network, Follow-Up Group. 'Neonatology, University Hospital of Zurich, Zurich, Switzerland; ${ }^{2}$ Developmental Center, University Childrens' Hospital Zurich, Zurich, Switzerland; ${ }^{3}$ Neonatology, University Hospital Zurich, Zurich, Switzerland; ${ }^{4}$ Neonatal and Pediatric Intensive Care Unit, Children's Hospital of Lucerne, Lucerne, Switzerland; ${ }^{5} \mathrm{CH}$, Switzerland

\subsection{6/archdischild-2014-307384.1071}

Background and aims In Switzerland, survival of extremely preterm infants (gestational age (GA) $<26$ weeks) improved following the publication of the 2002 Swiss Guidelines for the care of infants born at the limit of viability, and remained stable thereafter. We compared the 2-year-outcome of survivors of cohorts born before and after the publication of the Guidelines.

Methods 2-year-outcome of surviving preterm infants (GA 2225 weeks) born in 2000-2001 (pre-Guideline), 2003-2004 (post-Guideline), and 2005-2009 (long-term) were compared. Neurodevelopment was assessed using the BSID-II or the Griffiths Mental Development Scales. Moderate neurodevelopmental impairment (NDI) was defined as development index of -3/-2SD from the norm, or mild cerebral palsy (GMFCS level 2); severe NDI as developmental index of $<-3$ SD from the norm, cerebral palsy (GMFCS level $\geq 3$ ), deafness or blindness.

Results Of 342 survivors, follow-up data was available for 294 (86\%), 29 (8\%) infants were lost to follow-up, and 19 (6\%) datasets were not comparable. Normal development was observed in $48 \%, 47 \%$ and 54\%, moderate NDI in 25, 30 and $26 \%$, and severe NDI in $19 \%, 10 \%$ and $15 \%$ of the cases in the pre-Guideline cohort, the post-Guideline cohort and the longterm cohort, respectively. None of these differences over time were significant $\left(\chi^{2}, \mathrm{p}>0.1\right)$.

Conclusion 2-year-outcome in survivors after extremely preterm birth remained stable despite improved survival rates following the publication of the Guidelines for the care of infants born at the limit of viability.

\section{P0-0430 BRONCHOPULMONARY DYSPLASIA IS ASSOCIATED WITH DELAYED STRUCTURAL BRAIN MATURATION IN PRETERM INFANTS}

${ }^{1} \mathrm{~V}$ Neubauer, ${ }^{1} \mathrm{E}$ Griesmaier, ${ }^{2} \mathrm{D}$ Junker, ${ }^{2} \mathrm{M}$ Schocke, ${ }^{1} \mathrm{U}$ Kiechl-Kohlendorfer. ${ }^{1}$ Paediatrics II, Medical University Innsbruck, Innsbruck, Austria; ${ }^{2}$ Radiology, Medical University Innsbruck, Innsbruck, Austria

10.1136/archdischild-2014-307384.1072
Background and aims Cerebral MRI is increasingly used to depict the wide spectrum of preterm brain injury. Furthermore MRI also illustrates postnatal brain development in preterm infants. It has been shown by MRI at term-equivalent age (TEA) that preterm infants show a delayed brain maturation as compared to term infants and this delay has been related to neurobehavioral outcome. The aim of the present study was to investigate the influence of prevalent neonatal morbidities on structural cerebral brain maturation in a cohort of preterm infants born at a GA $\leq 32$ completed weeks.

Methods 130 very preterm infants born at a mean gestational age of $29.7 \pm 2.1$ weeks and a mean birthweight of $1265 \pm$ 405 grams were included. Brain MRI was performed at a mean postmenstrual age of 40.7 weeks (range 39-43). Structural brain development was evaluated by a validated "total maturation score". Brain maturation was correlated to neonatal data.

Results In univariate analysis bronchopulmonary dysplasia (BPD) and late-onset sepsis were significantly associated with delayed brain maturation. In multivariate analysis BPD remained significant for delayed maturation, with the diagnosis BPD carrying a fourfold risk for delay.

Conclusions This study is the first to show that delayed structural brain maturation in preterm infants at TEA is preceded by BPD, which is a known predictor of adverse outcome. This finding adds further evidence that the neural correlates of adverse outcome go beyond mere brain "injury".

\section{PO-0431 SERUM VASCULAR ENDOTHELIAL GROWTH FACTOR (VEGF) AND NEONATAL ENCEPHALOPATHY}

${ }^{1}$ TA Nuzum, ${ }^{1} \mathrm{R}$ Mooney, ${ }^{2} \mathrm{~S}$ Aslam, ${ }^{2} \mathrm{H}$ Eliwan, ${ }^{2} \mathrm{M}$ O'Hare, ${ }^{2} \mathrm{DU}$ Sweetman, ${ }^{2}$ EJ Molloy. ${ }^{1}$ Paediatrics, Royal College of Surgeons in Ireland, Dublin, Ireland; ${ }^{2}$ Neonatology, National Maternity Hospital Holles St., Dublin, Ireland

\subsection{6/archdischild-2014-307384.1073}

Background Pro-inflammatory cytokines in both cerebrospinal fluid (CSF) and serum have been associated with abnormal neurological findings following hypoxic-ischaemic injury. Vascular endothelial growth factor (VEGF) seems to have a neuroprotective effect in adult stroke and low levels correlate with poor outcomes. We aimed to establish a correlation between serum VEGF levels and the severity of Neonatal Encephalopathy (NE).

Methods Multi-organ evaluation was carried out on infants with NE $(n=122)$ with a particular emphasis on neurological status and function including neuroimaging (MRI and Cranial Ultrasound) and placental pathology. Serial serum VEGF levels were measured by multiplex ELISA over the first week of life.

Results Infants with NE $(n=73)$ had serum VEGF levels on days 1, 2 and 3 of life that decreased as the severity of $\mathrm{NE}$ increased (Grade 0: $\mathrm{n}=9$, Grade 1: $\mathrm{n}=20$, Grade 2: $\mathrm{n}=37$, Grade 3: $\mathrm{n}=7$ ).

Infants with $\mathrm{NE}(\mathrm{n}=122)$ had an association between elevated serum VEGF levels on day 1 and histological chorioamnionitis $(n=24)(p=0.025)$. The mean serum VEGF in those without chorioamnionitis was $570 \pm 870 \mathrm{pg} / \mathrm{mL}$ compared to $1144 \pm 1129 \mathrm{pg} / \mathrm{mL}$ in those with chorioamnionitis. Serial VEGF levels were significantly associated with cord anomalies $(\mathrm{p}<$ 0.05). VEGF did not correlate with MRI results.

Conclusions VEGF correlated with Histological Chorioamnionitis in NE and the severity of encephalopathy. 